\title{
Nucleosynthesis of s-elements in rotating AGB stars
}

\author{
L. Siess ${ }^{1}$, S. Goriely ${ }^{1}$, and N. Langer ${ }^{2}$ \\ 1 Institut d'Astronomie et d'Astrophysique, Université Libre de Bruxelles, CP 226, 1050 Brussels, Belgium \\ 2 Astronomical Institute, Utrecht University, The Netherlands
}

Received 5 September 2003 / Accepted 6 November 2003

\begin{abstract}
We analyze the s-process nucleosynthesis in models of rotating AGB stars, using a complete nuclear network covering nuclei up to Polonium. During the stage of thermal pulses, the extreme shear field that develops at the base of the convective envelope leads to the injection of protons into the adjacent ${ }^{12} \mathrm{C}$-rich core. Subsequent proton captures lead to overlapping ${ }^{14} \mathrm{~N}$-rich and ${ }^{13} \mathrm{C}$-rich layers. While the ${ }^{13} \mathrm{C}$ nuclei release neutrons due to $\alpha$-captures during the interpulse phase, the persistence of mixing due to differential rotation produces a contamination of the whole ${ }^{13} \mathrm{C}$-rich layer with ${ }^{14} \mathrm{~N}$. The result is a quenching of the s-process efficiency. Our study emphasizes the sensitivity of the s-process nucleosynthesis to the strength and duration of the shear mixing phase. Uncertainties in the rate of ${ }^{13} \mathrm{C}(\alpha, n)$ turn out to have small effects on the resultant distribution of s-elements. Finally, we show that in this framework, a deeper third dredge-up tends to further inhibit the production of s-elements.
\end{abstract}

Key words. nucleosynthesis - stars: AGB and post-AGB - nuclear reactions - abundances

\section{Introduction}

The principles of nucleosynthesis of s-elements are well understood (e.g. Clayton 1968): successive neutron captures on heavy nuclei, starting mostly from the Fe-group elements, are followed by beta decays, with a neutron capture time scale exceeding the half-lifes of most of the unstable nuclei. In AGB stars, the neutrons required for this process are mainly produced by $\alpha$-captures on ${ }^{13} \mathrm{C}$ in a thin radiative layer below the convective envelope. Although parametric studies, by artificially incorporating suitable amounts of ${ }^{13} \mathrm{C}$ into the system (Gallino et al. 1998, Busso et al. 2001) or by injecting protons into the C-rich layers at the time of the third dredge-up (Goriely \& Mowlavi 2000), are able to reproduce a large set of observational heavy element abundance data, the very origin of this so-called " ${ }^{13} \mathrm{C}$ pocket" remains the central problem of realistic s-process models. It has long been suggested (Iben \& Renzini 1982), that if protons from the envelope can mix with the carbon-rich layers left behind the pulse-driven convective shell, then ${ }^{13} \mathrm{C}$ can be synthesized through the chain of reaction ${ }^{12} \mathrm{C}(\mathrm{p}, \gamma){ }^{13} \mathrm{~N}\left(\beta^{+}\right){ }^{13} \mathrm{C}$. However, the physical mechanism responsible for injecting protons into the ${ }^{12} \mathrm{C}$-rich layers is still largely unknown.

The most recent attempts to identify the physical cause of this extra mixing refer to diffusive convective overshooting (Herwig et al. 1997; Goriely \& Mowlavi 2000), rotationally induced mixing (Langer et al. 1999; Herwig et al. 2003) or gravity waves (Denissenkov \& Tout 2003). The best studied mechanism so far is the overshoot model, which is able to produce s-process abundance patterns which are consistent

Send offprint requests to: L. Siess, e-mail: siess@astro.ulb. ac. be with many observations (Lugaro et al. 2003; Van Eck et al. 2003). However, some major problems still remain. In particular, the size of the partial mixing zone induced by overshooting - about $10^{-5} M_{\odot}$ - is one order of magnitude too small to explain observed barium surface overabundances of up to 100 in AGB stars (Goriely \& Mowlavi 2000). In addition, although the predictions of the overshooting scenario agree remarkably well with recent observations of low-metallicity $\mathrm{Pb}$ stars (Van Eck et al. 2001), they fail to reproduce another class of s-process-enriched low-metallicity stars that are depleted in $\mathrm{Pb}$ (e.g. Aoki et al. 2000, 2001, 2002; Van Eck et al. 2003). Finally, Van Winckel \& Reyniers (2000) have found a direct correlation between the total enrichment of s-process elements and the so-called [hs/ls] index (where hs refers to the heavy sprocess elements such as $\mathrm{Ba}, \mathrm{La}$ or $\mathrm{Ce}$, and ls to the light ones such as $\mathrm{Y}$ or $\mathrm{Zr}$ ). The overshoot model predicts an increase of the [hs/ls] ratio with decreasing metallicities, but not with the s-process enrichment, and once again fails to explain these new observations.

Therefore, further studies of the s-process in AGB stars are required. The present study focuses on the effects of rotation on the s-process nucleosynthesis. Our approach is based on stellar evolution models with rotation which include only a limited nuclear network, and post-processing calculations using a new code which couples the mixing and nucleosynthesis equations self-consistently for more than 500 nuclei, on about 200 discretized layers.

The plan of our paper is as follows: in the next section we describe the input physics of stellar evolution and postprocessing codes. Section 3 reviews the structure and evolution of our rotating AGB star models, in Sect. 4 we present 
and analyze the results of our post-processing nucleosynthesis calculations, and in Sect. 5 we draw conclusions.

\section{Numerical codes and input physics}

\subsection{Stellar evolution code}

The rotating stellar models used in this study are computed as in Langer et al. (1999), unless stated otherwise. In brief, we apply the Ledoux criterion for convection and semiconvection according to Langer et al. $(1983,1985)$. Thereby, mean molecular weight barriers are fully considered by our treatment of convection. The rotational physics we use is as described in Heger et al. (2000). Here, the effects of gradients of the mean molecular weight, which also pose barriers to the rotational mixing processes, are included as in Heger et al. 2000 (i.e., $f_{\mu}=0.05$ ).

The stellar evolution models are computed including a small nuclear network covering the pp-chains, the CNO cycles, and the major helium burning reactions (see Langer et al. 1999). OPAL opacities of Iglesias et al. (1992) are used.

\subsection{Post-processing code}

When the diffusion timescale of an isotope is comparable to its nuclear lifetime, a simultaneous solution of the diffusion and nucleosynthesis equations is needed. The key concern for the s-process is ${ }^{13} \mathrm{C}$ which is destroyed in a few thousand years, a duration comparable with the rotational mixing timescale. In this context, the system of equations to solve is

$$
\frac{\partial Y_{i}}{\partial t}=\text { nuclear terms }+\frac{\partial}{\partial m_{\mathrm{r}}}\left[\left(4 \pi r^{2} \rho\right)^{2} D \frac{\partial Y_{i}}{\partial m_{\mathrm{r}}}\right] \text {, }
$$

where $Y_{i}$ is the molar mass fraction of isotope $i$ and $D=$ $D_{\text {conv }}+D_{\text {rot }}$ is the sum of the Eulerian convective and rotational diffusion coefficients, respectively. After linearization of the equations, the solving the equations requires many inversions of a large matrix, the dimension of which is proportional to the number of considered isotopes and to the number of spatial grid points. To overwhelm exceedingly time consuming simulations inherent to the resolution of the fully coupled scheme, we have used the sophisticated DASPK package developed by the Livermore group which is based on the Krylov method (Brown et al. 1994, 1998). The great advantage of this implicit method is to significantly reduce the size of the matrix to be inverted, and to consequently speed up the calculations.

The nuclear physics input to the post-processing code is described in Goriely \& Mowlavi (2000) and Goriely \& Siess (2001). Briefly, the nuclear reaction network includes about 547 nuclei up to Po with all relevant nuclear $(\mathrm{n}-, \quad \mathrm{p}-, \alpha$-captures), weak (electron captures, $\beta$-decays) and electromagnetic (photodisintegration) interactions. Nuclear reaction rates are taken from the Nuclear Astrophysics Library of the Brussels University (available at http://www-astro.ulb.ac.be), which includes the latest experimental cross sections and calculated rates within the statistical Hauser-Feshbach model (Jorissen \& Goriely 2001). In particular, all the charged-particle-induced reaction rates of relevance in the $\mathrm{H}$ - and He-burning calculations presented here are taken from the European compilation of experimental rates, known as NACRE (Angulo et al. 1999).
The $\beta$-decay and electron capture rates in stellar conditions are taken from Takahashi \& Yokoi (1987) with the update of Goriely (1999).

In the post-processing code, the neutron abundance is set to its local equilibrium abundance and is decoupled from the diffusion equation. Since we focus on a very narrow mass region (a few $10^{-5} M_{\odot}$ ), boundary conditions have to be set carefully. On the uppermost layer, we impose the chemical abundances to be equal to the one computed by the stellar evolution code. Although the reaction rates are slightly different between the nuclear and stellar codes, the relatively low temperature in this upper limit of the integration zone does not affect the abundances determination which remains in very good agreement between the 2 codes. When the top of our numerical grid is located in the convective envelope, this last cell is treated as an infinite reservoir. Finally, at the bottom of the diffusion region, we allow matter to flow freely through the boundary. This is not very constraining since this region is chemically homogeneous as a result of the passage of the thermal pulse. The postprocessing calculations are started at the time of the third dredge-up (3DUP) and the initial chemical composition is given by the stellar evolution code. During the integration, a constant Lagrangian grid is imposed, and the thermodynamical quantities (temperature and density) are interpolated in the stellar models at the given time and mass coordinate. The mixing zone is typically described by 200 shells and encompasses $\sim 5 \times 10^{-5} M_{\odot}$.

\section{Structure and evolution of rotating AGB stars \\ 3.1. Evolutionary properties}

The initial model is a $3 M_{\odot}$ of solar chemical composition that was previously studied by Langer et al. (1999). Its initial rotation velocity was set to $v_{\text {init }}=250 \mathrm{~km} \mathrm{~s}^{-1}$ on the ZAMS, and the model is evolved up to the 29th thermal pulse (4 pulses later than in Langer et al. 1999).

The structural evolution of rotating AGB stars is described in detail in Herwig et al. (2003), and comparisons with nonrotating models can be found in Siess et al. (2003). As the He burning shell becomes thermally unstable, a convective zone develops that rapidly (in a few years) reaches the inner, fast rotating layers of the $\mathrm{CO}$ core. Angular momentum is dredgedup and removed from the central region, and redistributed over the convective zone. When the instability decays, the angular velocity of the contracting regions increases while the expanding shells slow down. Shortly thereafter, the slowly rotating convective envelope deepens and whenever the 3DUP occurs, a layer of strong differential rotation forms at the $\mathrm{H} /{ }^{12} \mathrm{C}$ discontinuity. In this region, the Goldreich-Schubert-Fricke (GSF) instability and to a lesser extent the secular shear instability and Eddington-Sweet circulations generate an efficient mixing which induces the transport of protons into the carbon rich region. Subsequent radiative hydrogen burning (Straniero et al. 1995) synthesizes the neutron seeds and forms the so-called ${ }^{113} \mathrm{C}$ pocket". The reason why GSF dominates (for part of the time) over Eddington-Sweet is that it depends strongly on the angular velocity gradient (which is strong in our case) and is not dependent on driving nuclear energy generation terms 


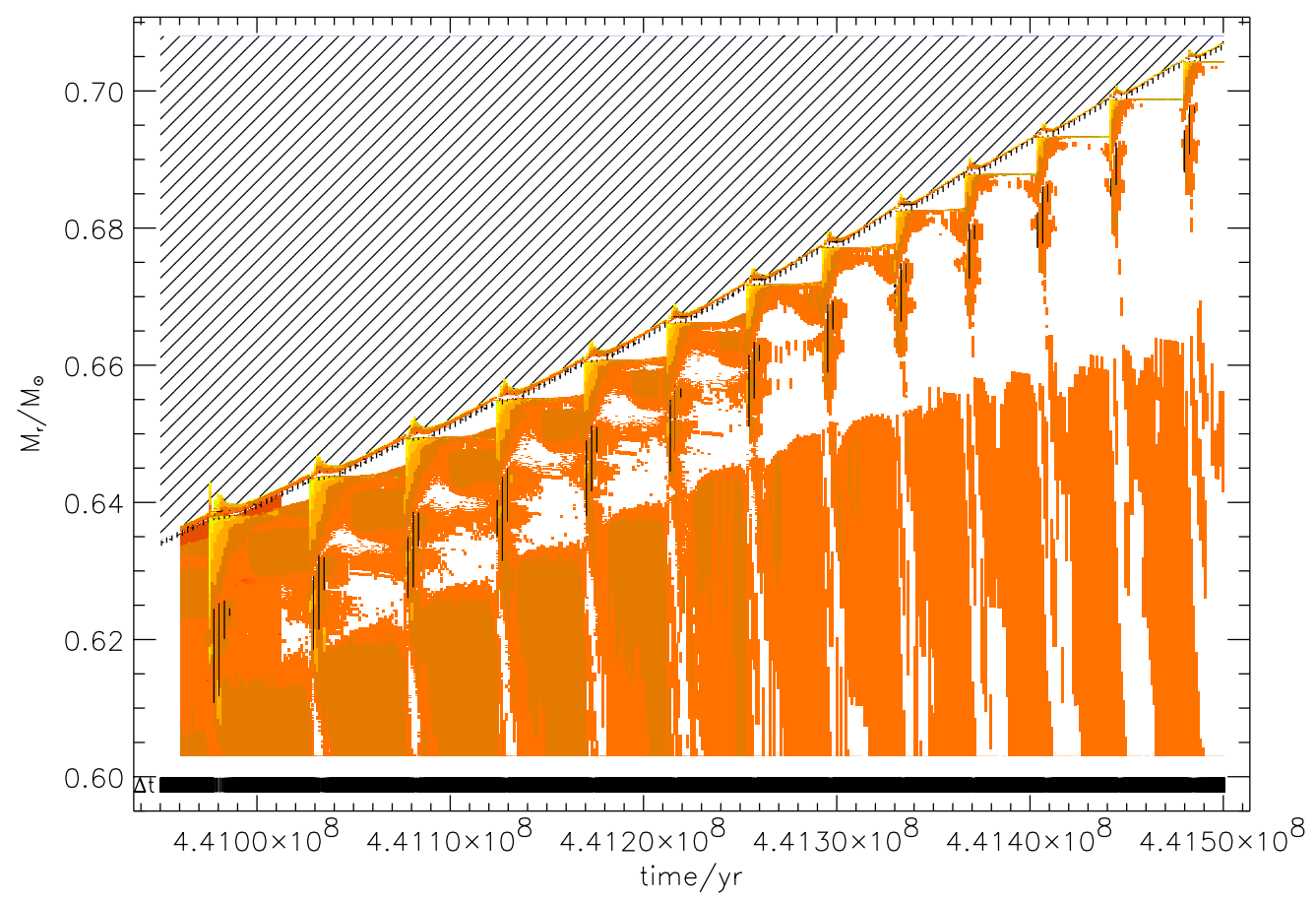

Fig. 1. Evolution of the internal structure of the rotating $3 M_{\odot}$ AGB model during 13 successive thermal pulses. Convective zones are represented by diagonal hatching, vertical hatching represents the nuclear burning regions, and grey coloring marks rotationally induced mixing, with lighter gray indicating faster mixing. In this model star, the 3DUP appears later, at pulse number 25 and our nucleosynthesis computations are made on pulse number 29 . The extent of the pulse driven-convective zones is typically of the order of $0.0080-0.0085 M_{\odot}$ and cannot be resolved on the figure. These instabilities last typically $\sim 100 \mathrm{yr}$ and soon after they decay, the bottom of the convective envelope recedes, as can be seen in the figure.

(which is weak after the He shell flash). It also dominates (partly) over the secular shear instability since its dependence on the mean molecular weight gradient (which slows down the mixing) is weaker.

The evolution of the internal structure during the first 13 pulses of the $3 M_{\odot}$ is depicted in Fig. 1 . We see that the size of the rotationally mixed intershell region decreases with time. Initially it extends over the entire intershell, and narrows to a thin band that is maintained during the whole interpulse phase. The reason for the decrease of the rotational mixing with time is to be attributed to the progressive removal of angular momentum from core by the successive thermal pulses and 3DUPs (Fig. 2). It appears that from pulse to pulse the angular momentum and the rotational velocity in the intershell region decrease. This braking of the central regions reduces the differential rotation and therefore the diffusion coefficient. It is thus expected that the efficiency and duration of rotational mixing will be significantly reduced in the late thermal pulses compared to what is obtained in these models.

As discussed in Langer et al. (1999) and Siess et al. (2003), the impact of the centrifugal force on the stellar structure is relatively weak, but the effects of rotationally induced chemical mixing can be substantial, as described in the next section.

\subsection{Dependence of the 3DUP on the parameters}

The development of the 3DUP, responsible for the formation of carbon stars, has long been a puzzle in AGB star modeling. Previous studies indicate that the occurrence and magnitude of this event depends on the stellar characteristics (such

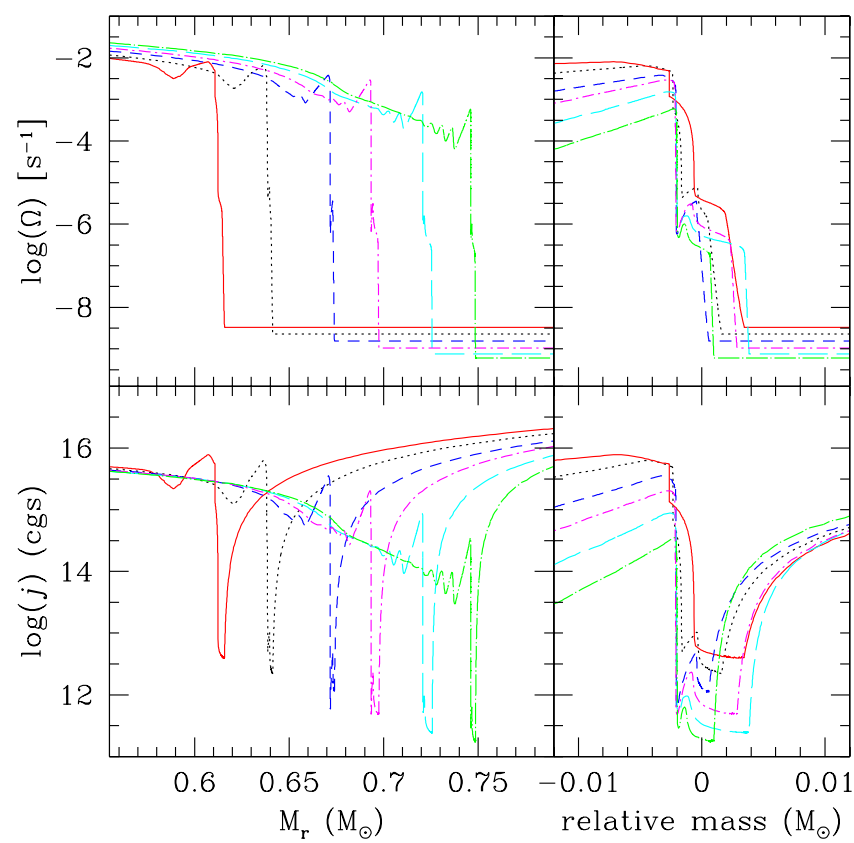

Fig. 2. Profiles of the local rotation frequency $(\Omega)$ and the specific angular momentum $(j)$ at different times during the interpulse phase of successive thermal pulses (cf. Fig. 1). By increasing mass, the profiles correspond to times (in $10^{8} \mathrm{yr}$ ) equal to $4.4074,4.4101,4.4127$, $4.4137,4.4172$ and 4.4174 , respectively. In the left panel, the same profiles are shown, but shifted in mass in order to be superposed, which we refer to as "relative mass" coordinate. The part of the $\Omega$-profile where $\Omega$ is constant belongs to the convective envelope. We note the progressive decrease in $\Omega$ and $j$ as the core mass increases. 
as mass, metallicity or mass loss rate), and on numerical factors associated with the treatment and resolution of convective boundaries (e.g. Frost \& Lattanzio 1996). It also became clear that extra-mixing favors the 3DUP as it smooths chemical gradients ( $\mu$-barrier) at the base of the convective envelope and allows the radiative and adiabatic gradient to reach neutrality $\left(\nabla_{\mathrm{rad}}=\nabla_{\mathrm{ad}}\right)$ at the convective boundary (e.g. Mowlavi 1999; Pols \& Tout 2000). Extra-mixing thus appears as the key process to understand the evolution and nucleosynthesis of AGB stars with substantial implications on the nucleosynthesis. One of the important parameter in our treatment of rotational mixing is $f_{\mu}$ (see Heger et al. 2000 for more details) which determines to what degree mean molecular weight barriers prevent or slow down the rotational mixing. Setting $f_{\mu}=0$, i.e. ignoring mean molecular weight barriers, enables a substantial 3DUP while increasing $f_{\mu}$ hampers the penetration of convective envelope into the intershell region (Siess et al. 2003). As emphasized by Heger et al. (2000), the calibration of this parameter is difficult since large uncertainties remain on this simplistic modeling of rotational mixing, and also because the conjugation of other factors, in particular the simultaneous interaction of various types of instabilities, may cause unaccounted effects. Therefore, we may use $f_{\mu}$ here simply as a parameter that allows us to analyze how the production of s-elements is affected by the depth of the 3DUP. In our models, increasing $f_{\mu}$ from 0 to 0.05 decreases the 3DUP parameter $\lambda$ from 0.53 to $\lambda=0.04$.

Changing the depth of the 3DUP modifies the mixing efficiency below the convective envelope during the entire interpulse phase thus affecting the creation of the ${ }^{13} \mathrm{C}$-rich layers and the subsequent s-process nucleosynthesis. As the envelope moves inward, it reaches regions of higher angular velocity. The differential rotation is thus larger when the 3DUP is deeper but on the other hand the mixing zone has moved into regions of smaller radii. As mentioned previously, the GoldreichSchubert-Fricke instability is the dominant mixing mechanism and its associated diffusion coefficient is proportional to $\Omega^{3} r^{5}$. Our results indicate that when the 3DUP is deeper, although $\Omega$ increases significantly (from $2.5 \times 10^{-7}$ to $1.4 \times 10^{-6} \mathrm{~s}^{-1}$ ), this effect is not compensated by the large decrease in radius (which passes from $4 \times 10^{10}$ to $7.5 \times 10^{9} \mathrm{~cm}$ ). The main consequence of a deeper 3DUP is thus a reduced diffusion coefficient which entails a less efficient mixing. Quantitatively, increasing $\lambda$ from 0.04 to 0.53 (i.e. decreasing $f_{\mu}$ from 0.05 to 0 ) decreases the diffusion coefficient by two orders of magnitude.

\section{S-process nucleosynthesis}

By nature, in the diffusive description of overshoot presented by Herwig et al. (1997) or in the new formalism based on the generation gravity waves by Denissenkov \& Tout (2003), mixing only develops at the edge of the convective regions. Therefore these mechanisms strongly depend on the spatial location of this boundary. In non-rotating AGB stars, protons are injected in the carbon-rich layers at the time of 3DUP. Later, when the convective envelope recedes, the chemical evolution of the mixed layer is the result of nuclear burning only since the transport mechanisms are not acting anymore in this region of the star. This characteristic is in strong contrast with the

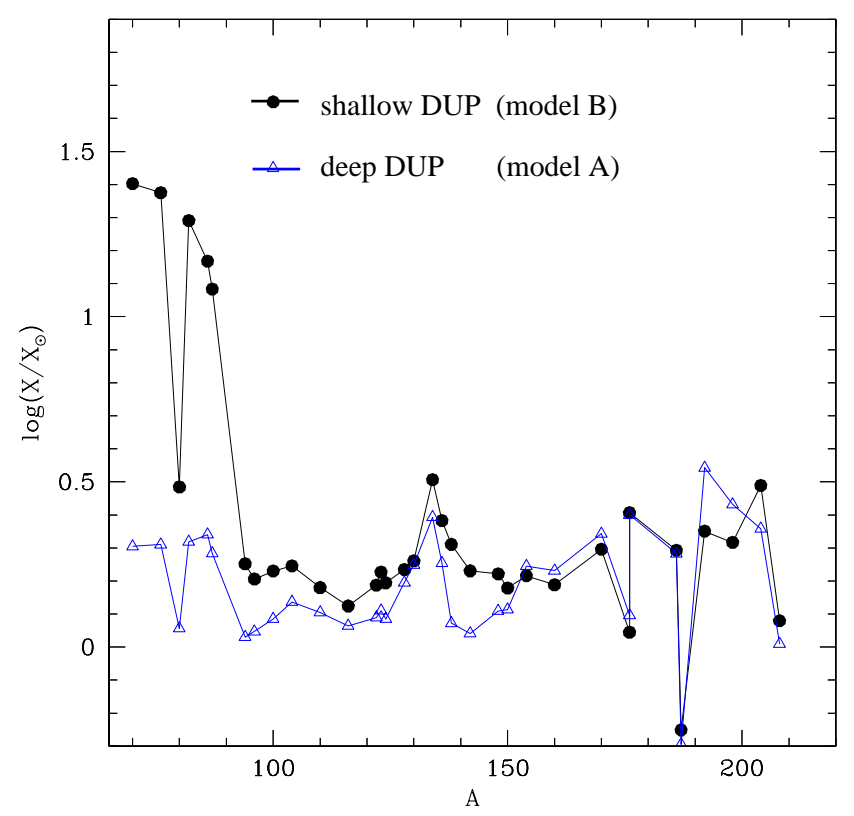

Fig. 3. Overproduction factors of s-only elements obtained for the $3 M_{\odot}$ rotating AGB models for different parameterization of the third dredge-up. The mass fraction of all species are summed over the same mass range and then normalized to solar.

rotationally induced mixing scenario. The strong differential rotation that establishes at the base of the convective envelope of AGB stars after the 3DUP sustains the transport of the chemical species during a substantial fraction of the interpulse period. The evolution of the ${ }^{13} \mathrm{C}$-rich layers is thus fundamentally different in this scenario.

The production of ${ }^{13} \mathrm{C}$ in the mixing layer depends on the proton-to-carbon abundance ratio $\left(Y_{\mathrm{p}} / Y_{{ }^{12} \mathrm{C}}\right)$. In the upper part of the region where protons are abundant, the $\mathrm{CN}$ cycle operates near equilibrium and leads mainly to the formation of a ${ }^{14} \mathrm{~N}$. Below this ${ }^{14} \mathrm{~N}$ layer, in a region where $10^{-2} \lesssim Y_{\mathrm{p}} / Y_{{ }^{12} \mathrm{C}} \lesssim$ $1,{ }^{13} \mathrm{C}$ is mostly produced. Consequently, the structure of the mixing region is stratified, consisting of a ${ }^{14} \mathrm{~N}$ layer on top of the ${ }^{13} \mathrm{C}$ pocket. Given the fundamental differences in the mixing scenarios mentioned above, we will see that the proximity of an efficient neutron poison $\left({ }^{14} \mathrm{~N}\right)$ to the neutron seeds $\left({ }^{13} \mathrm{C}\right)$ may quench the s-process nucleosynthesis if rotationally induced mixing acts after the formation of the ${ }^{14} \mathrm{~N}$ and ${ }^{13} \mathrm{C}$ layers and is efficient enough to mix them.

In the next section, we study how the different ingredients that influence mixing by rotation affect the production of s-elements.

\subsection{Depth of the 3DUP}

Figure 3 depicts the overproduction of s-only elements at the end of the interpulse phase of a $3 M_{\odot}$ model in two calculations involving a deep $\left(f_{\mu}=0\right.$, model $\left.\mathrm{A}\right)$ and a shallow $\left(f_{\mu}=0.05\right.$, model B) 3DUP characterized by $\lambda=0.53$ and $\lambda=0.04$, respectively. The distributions have been normalized to solar and show the quasi absence of s-elements in model A and a weak component in model $\mathrm{B}$, extending up to $A \sim 90$. The weakness of the s-process in both cases is the result of the transport of 


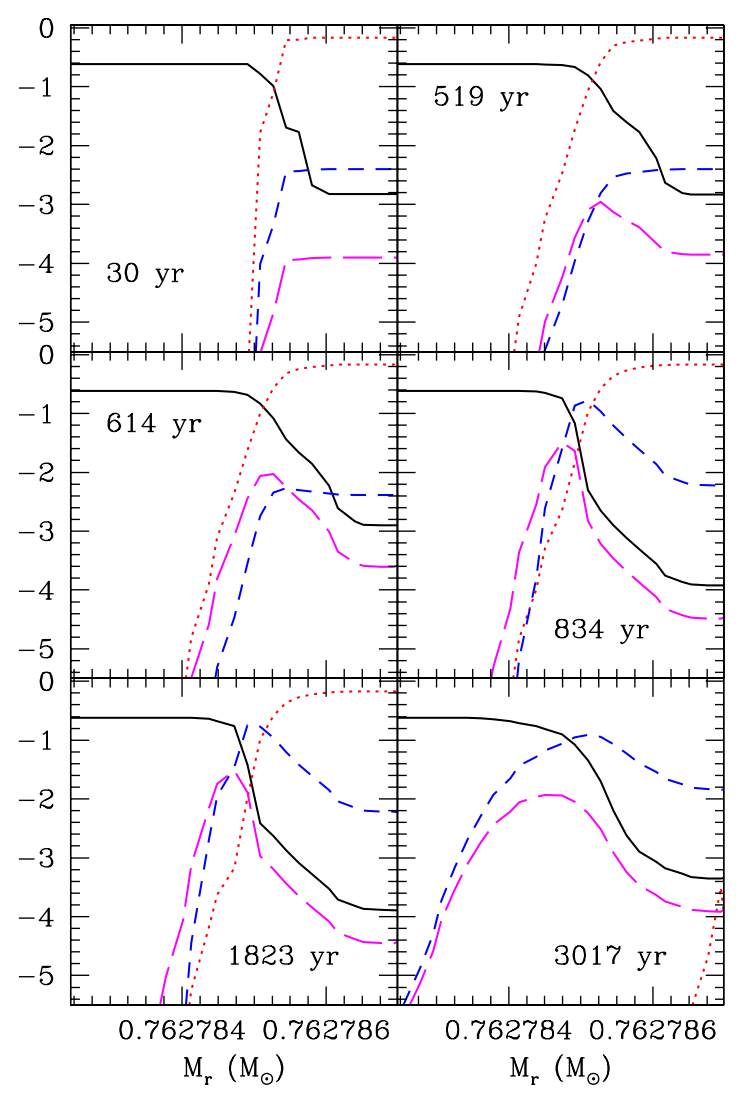

Fig. 4. Evolution of abundance profiles of ${ }^{12} \mathrm{C}$ (solid), $\mathrm{H}$ (dotted), ${ }^{13} \mathrm{C}$ (long-dashed) and ${ }^{14} \mathrm{~N}$ (short-dashed) in the mixing region of the $3 M_{\odot}$ model with $f_{\mu}=0$ (model A) after the 29 th pulse. The time is indicated in each panel and corresponds to the initial model just after the 3DUP (30 yr), the phase of diffusion and building up of a ${ }^{13} \mathrm{C}$ layer (519 and $614 \mathrm{yr}$ ), the phase of ${ }^{13} \mathrm{C}$ burning (834 and $1823 \mathrm{yr}$ ) and when the ${ }^{13} \mathrm{C}$-pocket is engulfed by ${ }^{14} \mathrm{~N}$ (3017 yr).

${ }^{14} \mathrm{~N}$ into the ${ }^{13} \mathrm{C}$-rich layers during the interpulse phase (Figs. 4 and 5). Because of its large neutron capture cross section, most of the released neutrons are captured by ${ }^{14} \mathrm{~N}$ rather than by iron seed nuclei and the production of s-elements is considerably weakened.

In model $\mathrm{A}$ the diffusion coefficient at the time of proton injection is 2 orders of magnitude smaller than in model B. The mass of the ${ }^{13} \mathrm{C}$-rich layer is about 4 times less massive and hence less neutrons are released. The contamination of the ${ }^{13} \mathrm{C}$-rich by ${ }^{14} \mathrm{~N}$ is also taking place in model $\mathrm{A}$ and contribute to further inhibits the s-process nucleosynthesis as illustrated in Figs. 4 and 5, which depict the evolution of the proton, ${ }^{12} \mathrm{C},{ }^{13} \mathrm{C}$ and ${ }^{14} \mathrm{~N}$ profiles in models $\mathrm{A}$ and $\mathrm{B}$. At the end of the interpulse phase, the neutron exposure is $\tau \sim 0.03$ and $0.09 \mathrm{mbar}^{-1}$ in case $\mathrm{A}$ and $\mathrm{B}$, respectively.

Surprisingly, the distribution of s-process elements in model B looks similar to that produced in the He-burning convective core of massive stars (e.g. Langer et al. 1989). The reasons are however different. In massive stars, efficient convective mixing does not allow heavy elements to be subject to successive neutron irradiations. The most abundant seeds to the s-process, i.e. the iron group elements, capture most of the neutrons, and this prevents the efficient nucleosynthesis of elements beyond $A \sim 90$. In our rotating AGB stars, ${ }^{14} \mathrm{~N}$ plays a

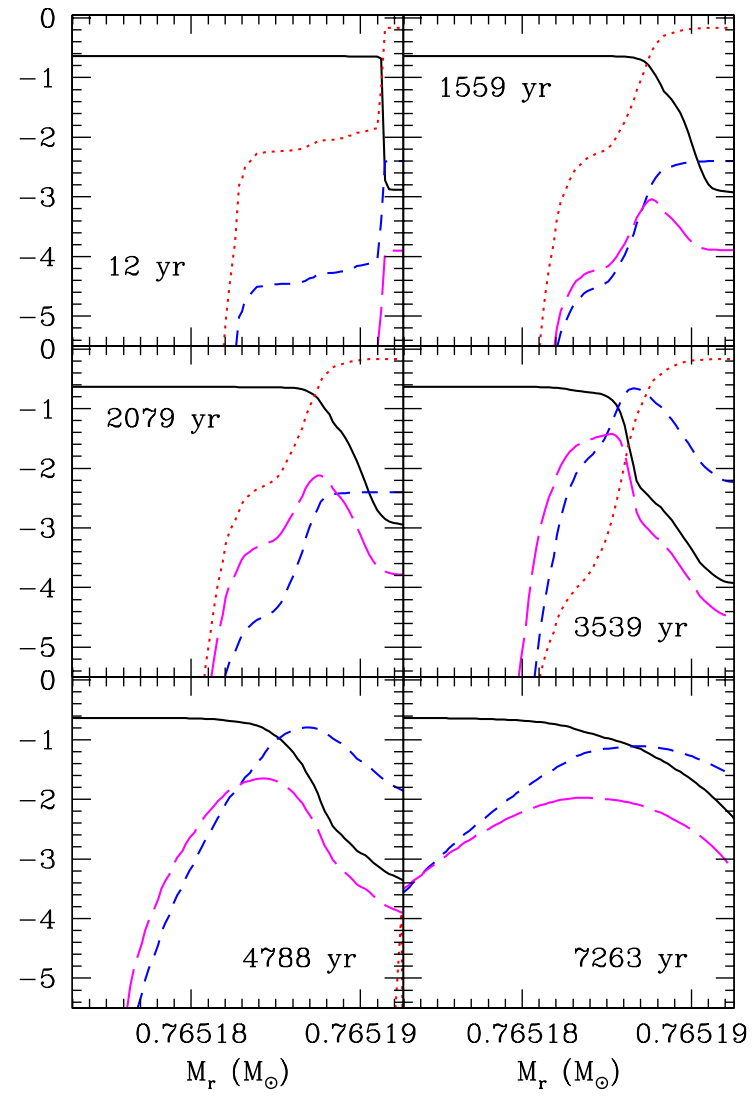

Fig. 5. Same as Fig. 4 but for model B with $f_{\mu}=0.05$. The mass interval between two big tick intervals is $10^{-6} M_{\odot}$. The non-zero initial abundances of protons and ${ }^{14} \mathrm{~N}$ in the ${ }^{12} \mathrm{C}$ region result from the appearance of a small convective zone below the convective envelope during the 3DUP.

similar role as the iron seeds, i.e., it limits the number of available neutrons and stops the s-process path at the magic neutron number $N=50(A=90)$. Another major difference between the present abundance pattern of s-only elements and that found during core He-burning of massive stars concerns the production of ${ }^{80} \mathrm{Kr}$. In contrast to massive stars where the neutron irradiation results from ${ }^{22} \mathrm{Ne}(\alpha, \mathrm{n})^{25} \mathrm{Mg}$ at temperatures typically of $3 \times 10^{8} \mathrm{~K}$, in the present scenario ${ }^{13} \mathrm{C}$ burns at temperatures of about $10^{8} \mathrm{~K}$ which makes the ${ }^{79} \mathrm{Se}$ temperature-dependent $\beta$-decay branching almost inexistent and does not enable the production of ${ }^{80} \mathrm{Kr}$.

\subsection{Mixing efficiency}

To investigate the impact of the mixing on the s-process nucleosynthesis, we arbitrarily increased or decreased the magnitude of the rotational diffusion coefficient and ran new simulations, using the same temperature and density profiles as in the standard case. Varying efficiencies of the mixing processes might be attributed to different initial rotational velocities, to the early occurrence of 3DUPs and early removal of angular momentum from the core, or to the effects of internal magnetic fields on the angular momentum distribution. In this approach, it is important to note that the structure and chemical profiles at the beginning of post-processing calculations are not entirely consistent, 


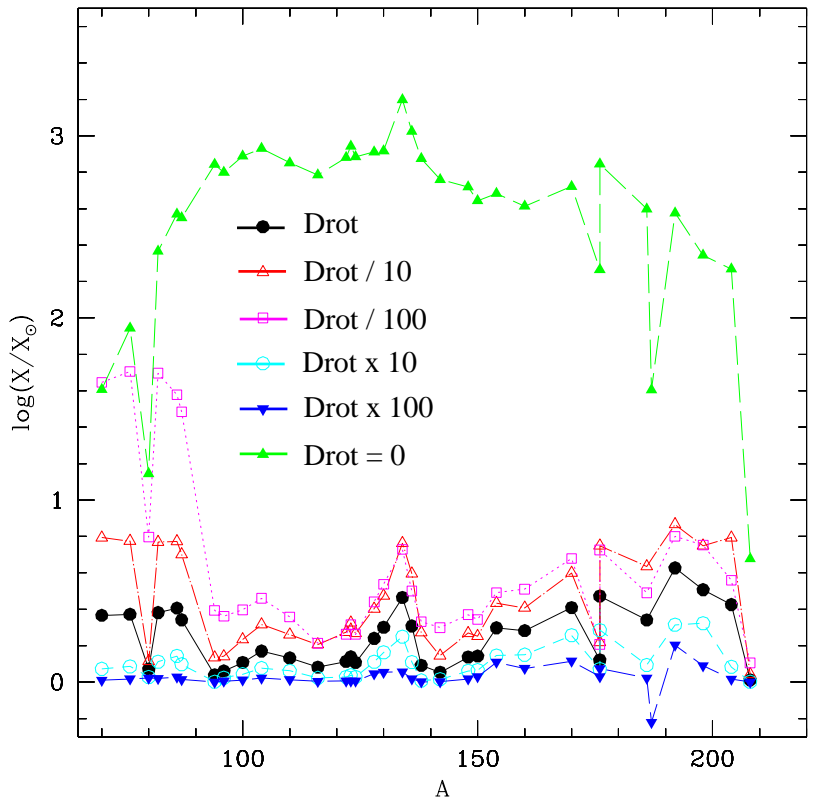

Fig. 6. Dependence of the overabundances of s-only elements on the diffusion coefficient. The stellar model used for these calculations is the $3 M_{\odot}$ models with a deep 3DUP (model A: $f_{\mu}=0$ ). The mass fraction of all species are summed over the same mass range and then normalized to solar. In the case $D_{\text {rot }}=0$, the s-process distribution results from our adopted initial conditions (see text).

since we did not re-compute the evolution of the star with these modified diffusion coefficients. In particular, the initial conditions are characterized by the fact that protons have already diffused in the underlying layers due to the deepening of the convective envelope. This is of no effects if the diffusion coefficient is increased since in this case the profiles will be modified anyway by the more efficient mixing. However when the diffusion coefficient is smaller, species that should not be there have already diffused below the envelope. We choose however to keep these initial conditions which in some ways mimic the effect of accounting for convective overshoot (see Sect. 4.3 and Fig. 8).

The results (Figs. 6 and 7) illustrate the great sensitivity of the s-process nucleosynthesis process to the rotational mixing. For small diffusion coefficients $\left(D_{\text {rot }} / 100\right)$ and provided some kind of mixing has taken place that can account for the initial proton profile, the pollution of the ${ }^{13} \mathrm{C}$-rich layers by ${ }^{14} \mathrm{~N}$ is marginal and the elemental distribution looks similar to the "pure" overshoot model (Fig. 8). On the other hand, for large values of $D_{\text {rot }}$, the ${ }^{13} \mathrm{C}$ pocket is rapidly contaminated by the abundant ${ }^{14} \mathrm{~N}$ and the s-process is inhibited. These conclusions appear to be independent of whether or not the star experiences a deep 3DUP.

For comparison, we ran a simulation using the timedependent diffusive approach of overshoot developed by Herwig et al. (1997). In this model, the diffusion coefficient below the convective envelope is given by

$D_{\text {over }}\left(r<r^{\text {env,bot }}\right)=D_{\text {conv }}^{\text {env,bot }} \times \mathrm{e}^{\frac{\left(r_{\text {env,bot }}^{\text {enr } r}\right)}{f_{\text {over }}^{\text {env, bot }}}}$

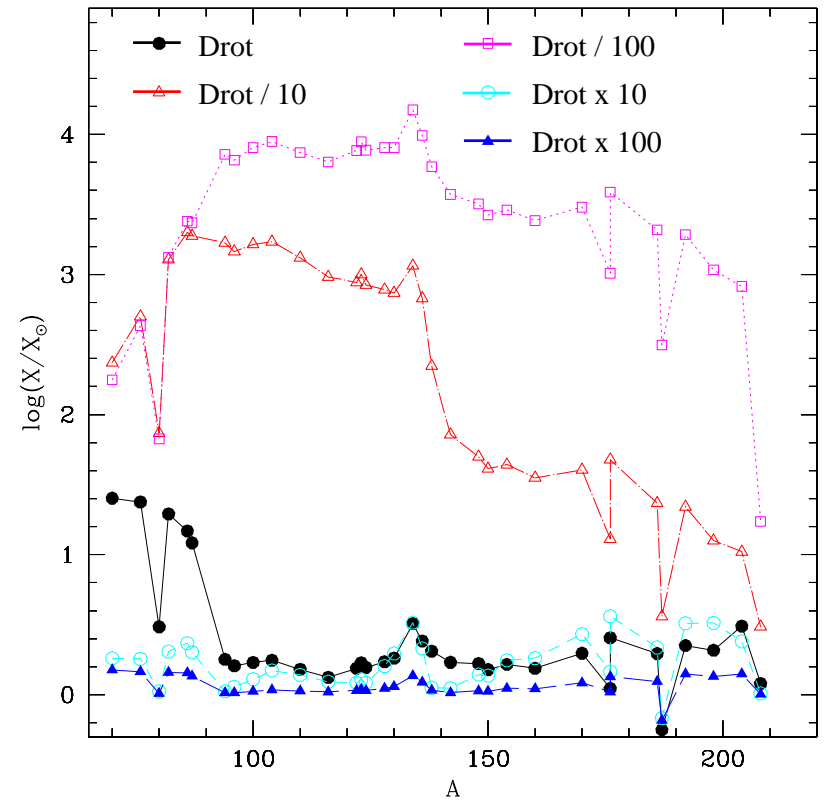

Fig. 7. Same as Fig. 6 but for model $\mathrm{B}\left(f_{\mu}=0.05\right)$.

where $r^{\text {env,bot }}$ and $H_{\mathrm{p}}^{\text {env,bot }}$ are the radius and pressure scale height at the bottom of the convective envelope, and $f_{\text {over }}$ is a free parameter equal to 0.1 in our test. In this framework, where rotational mixing is not included, once protons have polluted the carbon-rich layer, no more mixing takes place. The ${ }^{14} \mathrm{~N}$ layer remains disconnected from the ${ }^{13} \mathrm{C}$-rich layer and a strong s-process nucleosynthesis follows (Fig. 8).

A posteriori, the large observed dispersion in the distribution of s-elements could in principle be modulated by combining overshooting and rotational mixing, as proposed by Herwig et al. (2003). However, such a modulating effect due to rotation would still require modification of the rotation-induced mixing by about 2 decades (Figs. 6 and 7). Unless the rotational diffusion coefficient is strongly reduced by future improved descriptions (e.g. by including magnetic fields), rotation appears not to be able at this stage to solve the remaining observational puzzles, as discussed in Sect. 1. In addition the mass of the ${ }^{13} \mathrm{C}$ pocket obtained in these simulations $\left(\lessgtr 10^{-5} M_{\odot}\right)$ is still a factor of 10 smaller than what is required to reproduce the observations.

\subsection{Duration of mixing and evolution of the s-process distribution}

An important parameter arising from this study is the duration of the mixing process. In a series of computations, we artificially stop the rotational mixing at different epochs $\left(t_{\text {mix }}\right)$ to analyze how this mixing timescale influences the final distribution of s-elements (Fig. 8). Note that the profiles corresponding to the different $t_{\text {mix }}$ are shown in Fig. 4. In the first tests $\left(t_{\text {mix }}=834 \mathrm{yr}\right.$ and $\left.t_{\text {mix }}=1823 \mathrm{yr}\right)$, mixing is turned off after the ${ }^{13} \mathrm{C}$ pocket has formed: in these cases a large production of s-elements ensues since the ${ }^{13} \mathrm{C}$-rich layers are not or weakly contaminated by ${ }^{14} \mathrm{~N}$. The distribution at $t_{\text {mix }}=834 \mathrm{yr}$ is very similar to the case $t_{\mathrm{mix}}=0 \mathrm{yr}$ and the difference with 


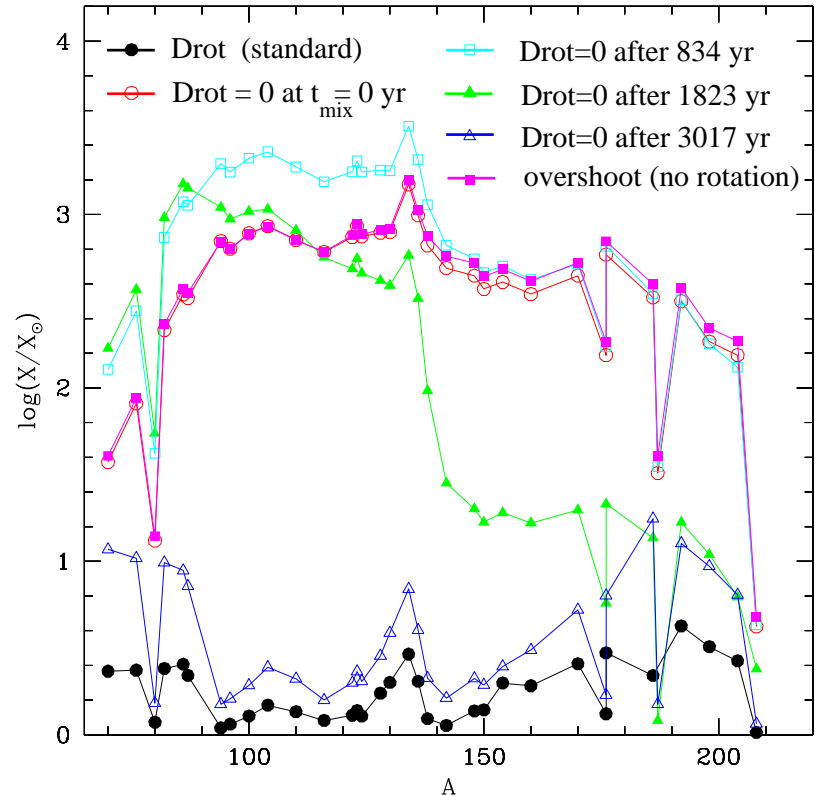

Fig. 8. Influence of the duration of rotational mixing on the overabundances of s-only elements for the $3 M_{\odot}$ models with a deep 3DUP (model A: $f_{\mu}=0$ ). The time $t_{\text {mix }}$ defined as the duration allowed for rotational mixing is labeled in the figure and the abundance profiles corresponding to these epochs are depicted in Fig. 4. For comparison, we also depict the distribution obtained with the overshoot model $\left(f_{\text {over }}=0.1\right)$ which superposes to the $D_{\text {rot }}=0$ case. The mass fraction of all species are summed over the same mass range and then normalized to solar except for the overshoot model where the distribution has been normalized to the $D_{\text {rot }}=0{ }^{124} \mathrm{Te}$ abundance, for comparison.

the case $t_{\mathrm{mix}}=1823 \mathrm{yr}$, found in the heavy s (hs) component, results from the fact that the partial diffusion of ${ }^{14} \mathrm{~N}$ reduces the number of neutrons to seeds ratio and consequently the sprocess efficiency. On the other hand, if rotationally induced mixing is maintained for a longer period of time, changing the magnitude of the rotational diffusion coefficient has very little impact, since here the s-process nucleosynthesis is anyway strongly inhibited. Finally, if rotational mixing is stopped once the convective envelope has reached its deepest extent (case $t_{\text {mix }}=0 \mathrm{yr}$ ), the s-abundance distribution is very similar to the one obtained with the overshoot model, indicating that rotational mixing gives similar initial conditions favorable for the s-process.

In rotating stars, chemical mixing results from the coupled effects of structural readjustments and angular momentum transport. Concentrating on this last point, we emphasize that our treatment of angular momentum transport does not include the advection term present in the more complete theory developed by Zahn (1992). This term has proved to be relevant during the main sequence of massive (Meynet \& Maeder 2000) and low-mass stars (Palacios et al. 2003, see their Fig. 5) especially in regions of strong gradients of mean molecular weight. In addition, in this region the Eddington Sweet circulation remains important at the base of the partial mixing zone. Since the advection term depends directly on the meridional velocity, in this context this effect might be relevant. Clearly this aspect deserves additional investigations.

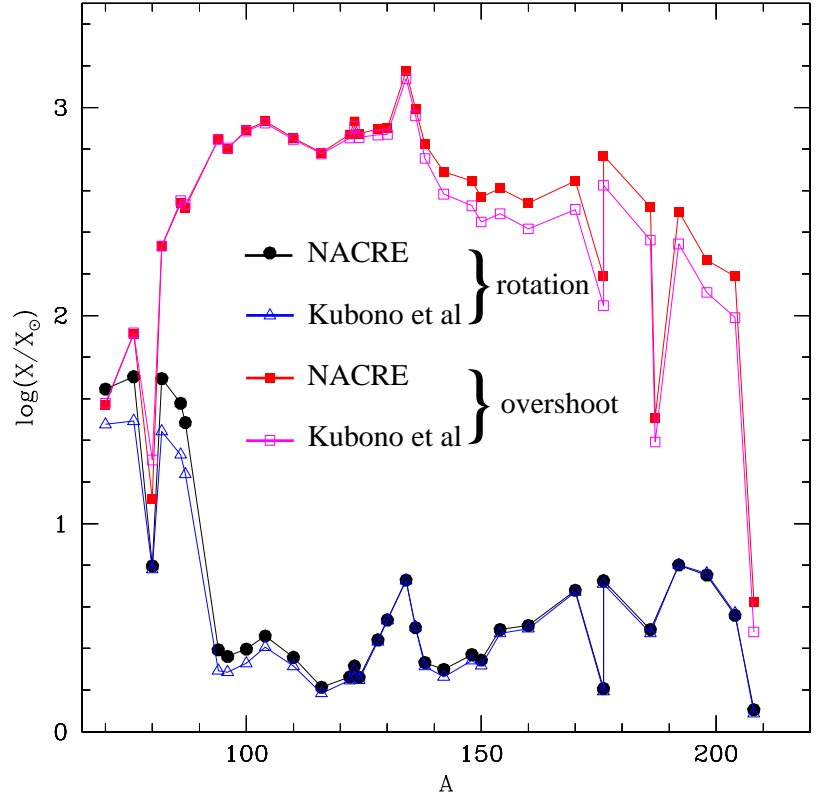

Fig. 9. Influence of ${ }^{13} \mathrm{C}(\alpha, \mathrm{n}){ }^{16} \mathrm{O}$ rate on the distribution of s-only elements. Overabundances are shown for 2 different mixing scenarios: rotational mixing with $D_{\text {rot }} / 100$ and overshoot model $\left(f_{\text {over }}=0.1\right)$.

Finally, we showed in Sect. 3 that as the core mass and dredge-up increase, the efficiency of mixing is reduced. In particular in Fig. 1 we clearly see that the duration and extent of the rotationally induced mixing decrease from pulse to pulse. In light of the results presented here, we may thus speculate that the production of s-elements could be enhanced in late thermal pulses and could be sensitive to the specific mass and metallicity of the star.

\subsection{Nuclear reaction rates}

The rate of the reaction ${ }^{13} \mathrm{C}(\alpha, \mathrm{n}){ }^{16} \mathrm{O}$ has been recently reexamined experimentally by Kubono et al. (2003) who find a smaller contribution to the subthresold state at $6.356 \mathrm{MeV}$ compared to the NACRE rate adopted here. This small contribution leads to a rate smaller by a factor of about 4 at $T_{8}=1$. In order to investigate the effects of this new rate on the production of s-elements, we computed a series of 2 runs based on our $3 M_{\odot}, f_{\mu}=0$ model: one test involves the overshoot model without rotation and the other includes rotational mixing only. In this latter case, we select the model where the diffusion coefficient is reduced by a factor of 100 in order to consider favorable weak s-process conditions. The results (Fig. 9) confirm that the slower rates proposed by Kubono et al. (2003) slightly decreases the efficiency of the s-process. The effects remain however relatively weak and do not affect our previous conclusions.

\subsection{Proton recycling}

The role of the proton recycling in the neutron production, as stressed by Herwig \& Langer (2001), is investigated in more detail in this section. The recycling takes place through the following sequence of reactions: the neutrons are initially 


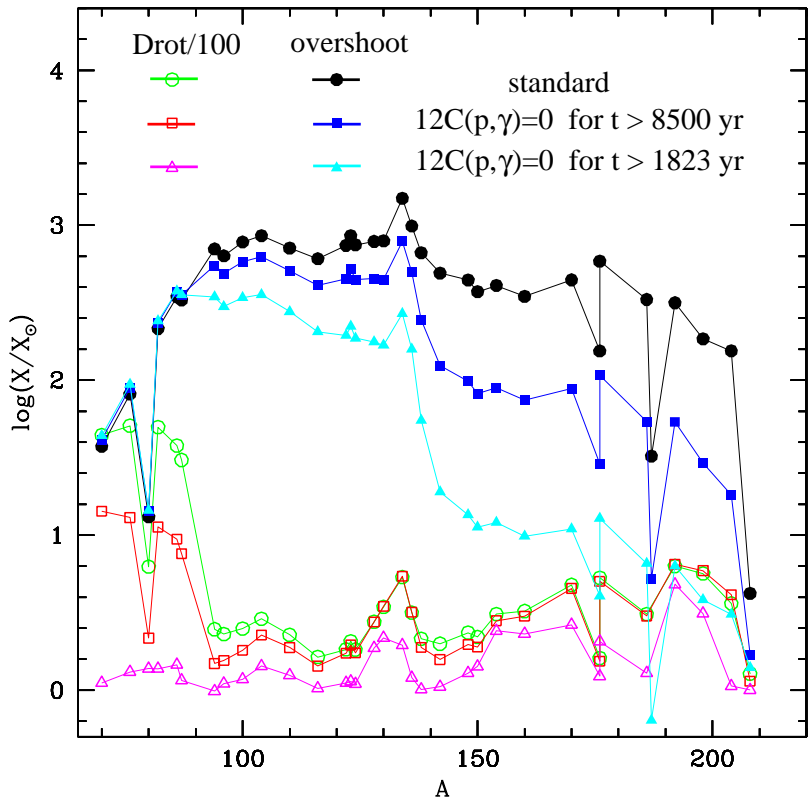

Fig. 10. Influence of proton-recycling on the nucleosynthesis of selements in rotating AGB stars. Overabundances are shown for 2 different models $\left(D_{\text {rot }} / 100\right.$ and the overshoot prescription with $f_{\text {over }}=$ $0.1)$ after the rate of ${ }^{12} \mathrm{C}(\mathrm{p}, \gamma)$ was set or not to zero.

produced by ${ }^{13} \mathrm{C}(\alpha, \mathrm{n}){ }^{16} \mathrm{O}$ and subsequently captured by the heavy elements to synthesize the s-elements but also by ${ }^{14} \mathrm{~N}$. Protons are then released by ${ }^{14} \mathrm{~N}(\mathrm{n}, \mathrm{p}){ }^{14} \mathrm{C}$ and can react with ${ }^{12} \mathrm{C}$ through ${ }^{12} \mathrm{C}(\mathrm{p}, \gamma){ }^{13} \mathrm{~N}\left(\beta^{+}\right){ }^{13} \mathrm{C}$ to regenerate the ${ }^{13} \mathrm{C}$ neutron seed. This proton source is also relevant to the production of ${ }^{19} \mathrm{~F}$ via the ${ }^{14} \mathrm{C}(\alpha, \gamma){ }^{18} \mathrm{O}(\mathrm{p}, \alpha){ }^{15} \mathrm{~N}$ chain of reactions followed by ${ }^{15} \mathrm{~N}(\alpha, \gamma){ }^{19} \mathrm{~F}$ later in the pulse-driven convective zone. To analyze the impact of this proton recycling on the s-process, we run a series of test computations where the rate of ${ }^{12} \mathrm{C}(\mathrm{p}, \gamma)$ is set to zero after the formation of the ${ }^{13} \mathrm{C}$-rich layer. The effect is depicted in Fig. 10 and indicates that in the rotational scenario without recycling, no s-elements are produced. If overshooting is used instead, the effects are smaller in part because in this case fewer ${ }^{14} \mathrm{~N}$ atoms are present in the region where neutrons are released. The tests also indicate that recycling is mostly efficient at the beginning of ${ }^{13} \mathrm{C}$ burning typically before $t \lesssim 10^{4} \mathrm{yr}$.

More generally, the conditions for an efficient proton recycling depend on several parameters and in particular on ${ }^{13} \mathrm{C}$ and ${ }^{14} \mathrm{~N}$ abundances, i.e the amount of neutron seeds and neutron poisons available. To estimate their impact, we perform parametric one-zone simulations for different initial ${ }^{13} \mathrm{C}$ and ${ }^{14} \mathrm{~N}$ abundances at a constant temperature $T=9 \times 10^{7} \mathrm{~K}$ and constant density of $\rho=10^{3} \mathrm{~g} \mathrm{~cm}^{-3}$, typical of those found in the radiative zones of AGB stars responsible for the s-process. The abundance of the other elements is taken as the one representative of the He-rich layers just below the convective envelope of our AGB model. These tests are conducted for two extreme initial ${ }^{12} \mathrm{C}$ abundances, namely the thermal pulse value of $X\left({ }^{12} \mathrm{C}\right)=0.2$ and the envelope value of $X\left({ }^{12} \mathrm{C}\right)=0.001$. The resulting time-integrated neutron exposure $\tau=\int N_{\mathrm{n}} v_{T} \mathrm{~d} t$ (where $v_{T}$ is the most probable relative neutron-nucleus velocity at the temperature $T$, and $N_{\mathrm{n}}$ is the neutron density)

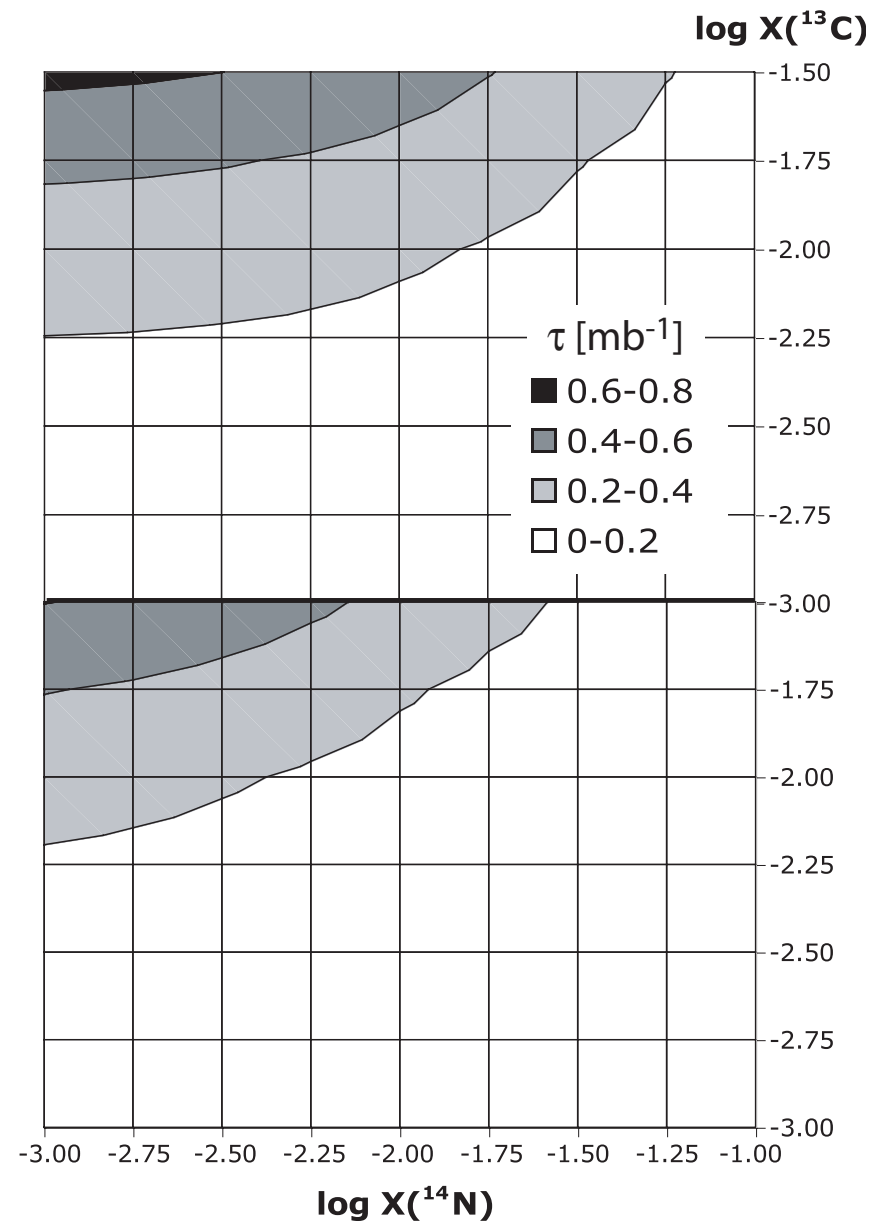

Fig. 11. Neutron exposure shown as contour plot for different initial ${ }^{13} \mathrm{C}$ and ${ }^{14} \mathrm{~N}$ abundances and for two different initial abundances of ${ }^{12} \mathrm{C}, X\left({ }^{12} \mathrm{C}\right)=0.2$ (upper panel) and $X\left({ }^{12} \mathrm{C}\right)=0.001$ (lower panel).

is displayed in Fig. 11. For a non-negligible s-process nucleosynthesis, the neutron exposure must reach at least $0.2 \mathrm{mb}^{-1}$. Comparing the upper and lower panels of Fig. 11 clearly shows the impact of the proton recycling by ${ }^{12} \mathrm{C}$ which mainly affects the s-process efficiency for $X\left({ }^{13} \mathrm{C}\right) \gtrsim 5 \times 10^{-3}$ and $X\left({ }^{14} \mathrm{~N}\right) \gtrsim$ $10^{-2}$. This regime is the one found in the rotationally-induced mixing zone before ${ }^{13} \mathrm{C}$-burning as illustrated in Figs. 4 and 5.

\section{Conclusion}

We have explored the effects of rotationally induced mixing on the nucleosynthesis of s-elements during the TP-AGB phase of a $3 M_{\odot}$. Our results show that rotation, while leading to the formation of a ${ }^{13} \mathrm{C}$-rich layer in the first place, quenches the s-process efficiency because of the contamination of the ${ }^{13} \mathrm{C}$ layer by ${ }^{14} \mathrm{~N}$, which confirms the recent findings of Herwig et al. (2003). While this result appears to be independent of whether or not the star experiences a deep 3DUP, the production of s-elements decreases further as the amount of 3DUP increases. We also show that the strength of the s-process depends strongly on the magnitude of the diffusion coefficient as well as on the duration of the interpulse mixing, the general 
trend being that longer periods of rotational mixing reduce the efficiency of the s-process.

Although we find that rotational mixing is an efficient mechanism to trigger the 3DUP, this process fails to reproduce the observed strong s-process overabundances. This does not mean that rotation is to be ruled out - intermediate mass stars are rapid rotators and significant amounts of shear at the bottom of the convective envelope in AGB stars are hard to avoid. However, it indicates that our treatment of rotation is not complete. As strong shear fields may lead to the generation of significant magnetic fields (Spruit 2003), it appears likely that a magnetic field will be generated in the vicinity of the partial mixing zone. The resulting magnetic torque may reduce the spin rate and thereby the rotational mixing. A first attempt to include magnetic fields in stellar evolution calculations has been performed by Heger et al. (2003), who showed that its presence can efficiently slow down the internal layers. In AGB stars, these effects could perhaps enable the initial injection of protons and the formation of the ${ }^{13} \mathrm{C}$ pocket but prevent the subsequent contamination of this layer by the poisonous ${ }^{14} \mathrm{~N}$. But these speculations require consistent simulations and a deep exploration of the parameter space.

Also, the presence of strong 3DUPs early in the TP-AGB phase could contribute to remove angular momentum from the core and may decrease the efficiency of mixing. In addition, large uncertainties still remain in this topics and in particular an investigation of the effects of metallicity would be very valuable. Decreasing metallicity tends to lower the effect of mean molecular weight barriers, with unknown consequences on the mixing episodes. Also the dependence of the induced mixing on the initial value of the rotational velocity $\left(\Omega_{\text {init }}\right)$ is largely unknown.

Acknowledgements. L.S. is grateful to Ana Palacios for fruitful discussions and acknowledges support from a European TMR "Marie Curie" fellowship at ULB. S.G. is FNRS Research Associate.

\section{References}

Aoki, W., Norris, J. E., Ryan, S. G., et al. 2000, ApJ, 561, L97 Aoki, W., Ryan, S. G., Norris, J. E., et al. 2001, ApJ, 561, 346 Aoki, W., Norris, J. E., Ryan, S. G., et al. 2002, ApJ, 580, 1149 Angulo, C., Arnould, M., Rayet, M., et al. 1999, Nucl. Phys. A, 656, 3

Brown, P. N., Hindmarsh, A. C., \& Petzold, L. R. 1994, SIAM J. Sci. Comp., 15, 1467
Brown, P. N., Hindmarsh, A. C., \& Petzold, L. R. 1998, SIAM J. Sci. Comp., 19, 1495

Busso, M., Gallino, R., \& Wasserburg, G. J. 1999, ARA\&A, 37, 239

Busso, M., Gallino, R., Lambert, D. L., Travaglio, C., \& Smith, V. V. 2001, ApJ, 557, 802

Clayton, D. D., in Principle of stellar evolution and nucleosynthesis, (New-York: McGraw-Hill), 1968

Denissenkov, P. A., \& Tout, C. A. 2003, MNRAS, 340, 722

Frost, C. A., \& Lattanzio, J. C. 1996, ApJ, 473, 383

Gallino, R., Arlandini, C., Busso, M., et al. 1998, ApJ, 497, 388

Goriely, S. 1999, A\&A, 342, 881

Goriely, S., \& Mowlavi, N. 2000, A\&A, 362, 599

Goriely, S., \& Siess, L. 2001, A\&A, 378, L25

Heger, A., Langer, N., \& Woosley, S. E. 2000, 528, 1033

Heger, A., Woosley, S. E., Langer, N., \& Spruit, H. C. 2003, to appear in Proc. IAU 215 Stellar Rotation

Herwig, F., Blöcker, T., Schönberner, D., \& El Eid, M. 1997, A\&A, 324, L81

Herwig, F., Blöcker, T., \& Schönberner, D. 1999, in 191st IAU Symp. (PASP), ed. T. Le Bertre, A. Lebre, \& C. Waelkens, 41

Herwig, F., \& Langer, N. 2001, Mem. Soc. Astron. It., 72, 277

Herwig, F., Lugaro, M., \& Langer, N. 2003, ApJ, 593, 1056

Iben, I. Jr., \& Renzini, A. 1982, A\&A, 263, L23

Iglesias, C. A., Rogers, F. J., \& Wilson, B. G. 1992, ApJ, 397, 717

Jorissen, A. S., \& Goriely, S. 2001, Nucl. Phys. A, 688, 508

Kubono, S., Abe, K., Kato, S., Teranishi, T., Kurokawa, M., \& Liu, X. 2003, Phys. Rev. Lett., 90, 062501

Langer, N., Fricke, K. J., \& Sugimoto, D. 1983, A\&A, 126, 207

Langer, N., El Eid, M. F., \& Fricke, K. J. 1985, A\&A, 145, 179

Langer, N., Arcoragi, J. P., \& Arnould, M. 1989, A\&A, 210, 187

Langer, N., Heger, A., Wellstein, S., \& Herwig, F. 1999, A\&A, 346, L37

Lugaro, M., Herwig, F., Lattanzio, J., Gallino, R., \& Straniero, O. 2003, ApJ, 586, 1305

Meynet, G., \& Maeder, A. 2000, A\&A, 361, 101

Mowlavi, N. 1999, A\&A, 344, 617

Palacios, A., Talon, S., Charbonnel, C., \& Forestini, M. 2003, A\&A, 399, 603

Pols, O. R., \& Tout, C. A. 2000, Mem Soc. Ast. It., 71, 753

Siess, L., Goriely, S., \& Langer, N. 2003, PASA, 20, 371

Spruit, H. C. 2003, A\&A, 381, 923

Straniero, O., Gallino, R., Busso, M., et al. 1995, ApJ, L440

Takahashi, K., \& Yokoi, K. 1987, At. Data Nucl. Data Tables 36, 375

Van Eck, S., Goriely, S., Jorissen, A., \& Plez, B. 2001, Nature, 412, 793

Van Eck, S., Goriely, S., Jorissen, A., \& Plez, B. 2003, A\&A, 404, 291

Van Winckel, H., \& Reyniers, M. 2000, A\&A, 354, 135

Zahn, J.-P. 1992, A\&A, 265, 115 